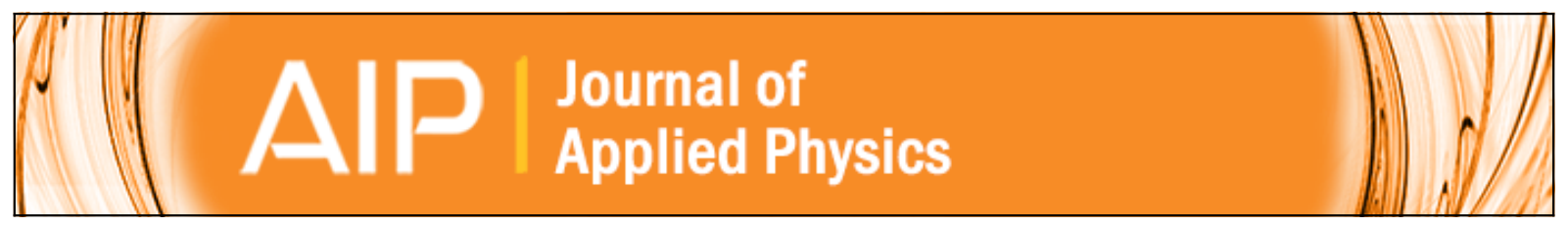

Strong dependence of the Fe thin-film magnetic anisotropy on the $\mathrm{Si}(111)$ substrate preparation

M. Cougo dos Santos, J. Geshev, D. K. Silva, J. E. Schmidt, L. G. Pereira, R. Hubler, and P. Allongue

Citation: Journal of Applied Physics 94, 1490 (2003); doi: 10.1063/1.1588363

View online: http://dx.doi.org/10.1063/1.1588363

View Table of Contents: http://scitation.aip.org/content/aip/journal/jap/94/3?ver=pdfcov

Published by the AIP Publishing 


\title{
Strong dependence of the Fe thin-film magnetic anisotropy on the Si(111) substrate preparation
}

\author{
M. Cougo dos Santos, ${ }^{\text {a) }}$ J. Geshev, D. K. Silva, J. E. Schmidt, and L. G. Pereira \\ Instituto de Física-UFRGS, C.P. 15051, 91501-970 Porto Alegre, RS, Brazil \\ R. Hubler \\ Puntifícia Universidade Católica-PUC, C.P. 1429, 90619-900 Porto Alegre, RS, Brazil \\ P. Allongue \\ Laboratoire Physique des Liquides et Électrochimie (CNRS UPR-15), Université P \& M Curie, \\ 4 Place Jussieu, Tour 22, F-75005 Paris, France
}

(Received 27 December 2002; accepted 8 May 2003)

\begin{abstract}
We report on the influence of the $\mathrm{Si}(111)$ surface preparation on both the structural and the magnetic properties of iron thin films grown onto them. Two different substrate preparation procedures were used, in one of which the substrate was purposely oxidized. The surface analysis was performed by using atomic force and scanning tunneling microscopies, and the magnetic behavior of the films was observed by magneto-optic Kerr effect polarimetry. A strong relationship between the substrate preparation procedure, morphology, and the magnetic response of the iron films was found. A phenomenological model was used to interpret the experimental magnetization data and to derive the anisotropy parameters for both types of Fe samples. It was found that the magnetic response of the film grown onto the oxidized substrate is dominated by the uniaxial anisotropy originated from the substrate topology (a terracelike structure); the behavior of the other film, grown onto the unoxidized substrate, is determined by both cubic and easy-plane anisotropies. (C) 2003 American Institute of Physics. [DOI: 10.1063/1.1588363]
\end{abstract}

\section{INTRODUCTION}

Substrate preparation conditions play an important role in the growth of magnetic materials and on the generation of sophisticated magnetic nanostructures. ${ }^{1-3}$ In particular, the surface preparation of $\mathrm{Si}(111)$ by itself is an actively pursued area of research, especially as a means of generating periodically ordered structures to be used as templates. Recently, Itaya et $a l .{ }^{4}$ demonstrated that etching $\operatorname{Si}(111)$ surfaces in neutral to basic ammonium fluoride (AF) solutions leads to the formation of atomically flat terracelike structures. Other works have shown the formation of triangular etched pits, ${ }^{5,6}$ findings obtained from the analysis of the initial stages of oxidation of $\mathrm{Si}$ surfaces in $\mathrm{AF}$ solutions and that have not been Ar sparged to eliminate the presence of the oxygen.

Wang and $\mathrm{Chen}^{7}$ and Cheng et al. ${ }^{8}$ have found that $\alpha$-Fe is formed when iron thin films are deposited on $\mathrm{Si}(111)$ at room temperature; the formation of twins ${ }^{9}$ during the epitaxial growth in these $\alpha$-Fe films has been observed.

Several studies on epitaxial Fe films, and on selfassembled $\mathrm{Fe}$ nanostructures, grown onto $\mathrm{Si}(111)$ substrates have been reported ${ }^{10,11}$ where it has been shown that the silicon surface morphology can induce very unique magnetic behavior on these deposits. Our recent works have demonstrated that the observed negative remanent magnetization in such a system can be attributed to a very small misorientation of the Fe layer from the (111) plane and/or to competing anisotropy effects. ${ }^{11,12}$

${ }^{\text {a)} E l e c t r o n i c ~ m a i l: ~ c o u g o @ i f . u f r g s . b r ~}$
The aim of the present study was to investigate the relationship between the AF-etched $\mathrm{Si}(111)$ substrates preparation conditions (with or without subsequent controlled oxidation), the morphology of iron films grown onto them, and their magnetic behavior.

This was done for two different samples: In one of them, the Fe was deposited onto a bare AF-etched substrate, and the other was deposited onto an AF-etched and subsequently oxidized substrate.

\section{EXPERIMENT}

The substrates used were $n$-type $\operatorname{Si}(111)$ wafers, with resistivities in the range of $2-3 \Omega \mathrm{cm}$. The samples were initially cleaned in two volumes of sulfuric acid (Merch, analytical grade) and 1 volume of $30 \%$ by weight aqueous hydrogen peroxide (Merck, analytical grade), for $20 \mathrm{~min}$ at $100^{\circ} \mathrm{C}$. The wafers were rinsed with $18 \mathrm{M} \Omega \mathrm{cm}$ water before and after each cleaning step. The clean silicon wafers were then immersed and held vertically in AF solutions contained in Teflon vials, and sparged with argon for 20 min via a Teflon tube inserted into the solution. A $40 \%$ by weight aqueous AF solution (Carlo Erba, clean-room grade) was used. Then two distinct procedures were used:

(1) One of the etched Si substrates was kept in nonoxidizing atmosphere, here named "bare" $\mathrm{Si}$; and

(2) The other was maintained in air for $24 \mathrm{~h}$ in order to produce an oxidized substrate, here named "oxidized" Si. 

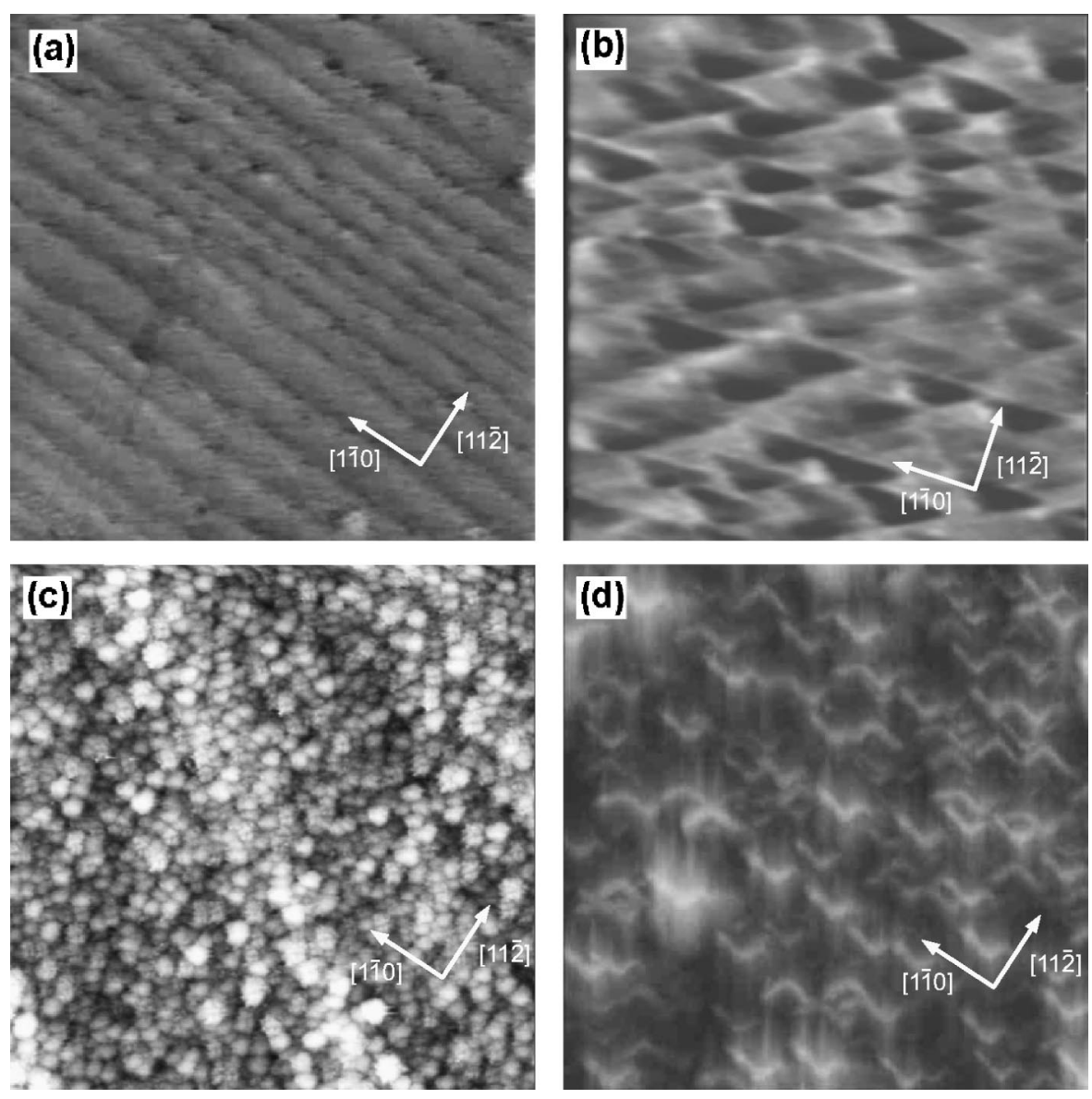

FIG. 1. $400 \mathrm{~nm} \times 400 \mathrm{~nm}$ AFM and STM images. (a) AFM image of $\mathrm{H}-\mathrm{Si}(111)$ after 20 min immersion in $\mathrm{NH}_{4} \mathrm{~F}$ solution; (b) AFM substrate surface image after $24 \mathrm{~h}$ exposure to moist air; (c) and (d) STM images after the growth of $6 \mathrm{~nm}$ thick iron layers onto the substrates shown in (a) and (b), respectively.
Subsequently, Fe films with a thickness of $t=6 \mathrm{~nm}$ were sputtered onto both substrates.

The iron films were deposited by rf sputtering in a 3.2 $\times 10^{-4}$ Torr Ar atmosphere for a base pressure before depositing lower than $10^{-8}$ Torr, and a deposition rate less than 1 $\AA /$ s. Both substrate preparation and Fe deposition were done at room temperature. The surface analysis was performed via atomic force microscopy (AFM) and scanning tunneling microscopy (STM) using a Digital Instruments Nanoscope IIIa.

The magnetic properties of the samples were characterized by an ex situ magneto-optic Kerr effect polarimetry. The instrument consists of a linearly polarized diode laser source with $5 \mathrm{~mW}$ output (operating at 638-680 nm), two polarizers, and a photodiode system. Measurements in the transversal geometry were performed with the magnetic field, $\mathbf{H}$, applied along different directions in the plane of the sample and perpendicular to the plane of incidence of the light. The angle of incidence was maintained at $60^{\circ}$ with respect to the normal direction of the film.

\section{RESULTS AND DISCUSSION}

Figure 1 shows the morphology of the bare Si and of the oxidized Si substrates, as well those of the Fe films deposited onto them, respectively. Panel (a) in Fig. 1 shows the AFM image of the bare $\mathrm{Si}$ substrate, where large terraces with uniform step distribution can be observed. The mean step height is approximately $3.2 \AA$, which corresponds to a double $\mathrm{Si}$ atomic layer. In contrast, the AFM image of the oxidized Si substrate [panel (b) in Fig. 1] shows different but expected standard triangular-shaped pits. ${ }^{6}$

The STM images of the Fe deposits, shown in Figs. 1(c) and 1(d), demonstrate that the different preparation conditions of the substrates result in very different iron growth modes. Clearly, a random distribution of grains with a mean width equal to $(8.6 \pm 0.6) \mathrm{nm}$ and height of $(6.0 \pm 0.5) \mathrm{nm}$ is observed in the image of the bare Si substrate [(Fig. 1(c)]. Cheng et al. ${ }^{8}$ have also observed grains about $10 \mathrm{~nm}$ in width aligned along the [111] direction in their epitaxial $\alpha$-Fe thin films grown onto $\mathrm{Si}(111)$. The growth mode for the sample sputtered onto the oxidized Si substrate is completely different. Formation of self-assembled $120^{\circ}$ wing-shaped islands is observed, i.e., this oxidized substrate creates a pattern for a self-organized iron growth. Our previous work ${ }^{11}$ reported that iron thin films grown on vicinal $\mathrm{Si}(111)$ treated with a HF solution showed a very clear striped structure. The absence of the latter in the present case does not necessarily mean that the Si surface is exactly the (111) plane. Itaya et $a l .{ }^{4}$ showed that striped structures are observed when the $\mathrm{Si}$ surface is treated with HF solution; in contrast, very flat $\mathrm{Si}$ surfaces are obtained if they are treated with $\mathrm{NH}_{4} \mathrm{~F}$ solution.

The magnetization measurements showed that the hysteresis loops, measured for various in-plane field directions for the sample grown onto bare $\mathrm{Si}$, are practically identical, which means that this system is magnetically isotropic. A representative hysteresis loop is plotted in panel (a) of Fig. 2. 


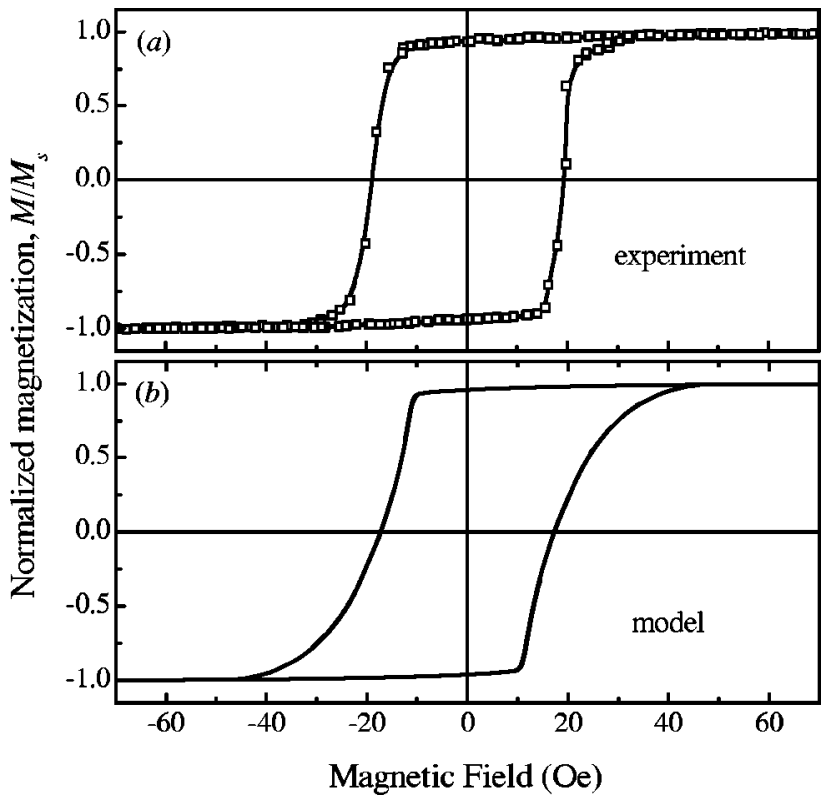

FIG. 2. (a) Representative experimental in-plane hysteresis loop for the sample whose surface topology is illustrated in Fig. 1(c) (the line is only a guide for the eyes); (b) Calculated curve for a system of noninteracting (111)-textured particles with random orientation in the film plane with a shape of an oblate spheroid with height-to-width ratio equal to $0.68, M_{s}$ $=1708 \mathrm{emu} / \mathrm{cm}^{3}, \quad K_{1}=4.80 \times 10^{5} \mathrm{erg} / \mathrm{cm}^{3}, \quad K_{2}=2.56 \times 10^{4} \mathrm{erg} / \mathrm{cm}^{3}$, and $K_{u}=0$.

Panel (b) in Fig. 2 shows the corresponding hysteresis loop calculated using a phenomenological model; the details of the model and the discussion of the fittings curves are given next.

Figure 3 shows three representative hysteresis loops, for $\phi_{H}=0^{\circ}, 55^{\circ}$, and $80^{\circ}$ for the Fe sample grown onto the oxidized $\mathrm{Si}$ substrate, where $\phi_{H}$ is the angle between the positive field direction and the in-plane easy magnetization axis, and $M$ and $M_{s}$ are the magnetization and saturation magnetization of the sample, respectively. As can be seen, its behavior is characteristic of a system with in-plane uniaxial anisotropy, where the loops range from almost rectangular (measured along the direction of one of the wings of the wing-shaped islands), to almost anhysteretic, low remanence (e.g., for $\phi_{H}=80^{\circ}$ ) curves.

The phenomenological model used to interpret the experimental data, plotted in Figs. 2(a) and 3, takes into consideration a ferromagnet whose anisotropy has cubic magnetocrystalline and uniaxial components; its magnetization vector $\mathbf{M}_{\mathbf{s}}$ is characterized by the direction cosines $\alpha_{1}, \alpha_{2}$, and $\alpha_{3}$, referred to the cube axes. For fixed magnitude and direction of $\mathbf{H}$, neglecting the thermal activation effects and considering only coherent magnetization rotation, the total free energy per volume $E$ can be written as

$$
\begin{aligned}
E= & K_{1}\left(\alpha_{1}^{2} \alpha_{2}^{2}+\alpha_{2}^{2} \alpha_{3}^{2}+\alpha_{3}^{2} \alpha_{1}^{2}\right)+K_{2} \alpha_{1}^{2} \alpha_{2}^{2} \alpha_{3}^{2} \\
& -K_{u}\left(\mathbf{M}_{\mathrm{s}} \cdot \mathbf{u} / M_{s}\right)^{2}-K_{d}\left(\mathbf{M}_{\mathrm{s}} \cdot \mathbf{n} / M_{s}\right)^{2}-\mathbf{M}_{\mathrm{s}} \cdot \mathbf{H},
\end{aligned}
$$

where $K_{1}$ and $K_{2}$ are the first two cubic anisotropy constants, $K_{u}$ is the uniaxial anisotropy constant, and the last two terms refer to the demagnetization energy (with constant $K_{d}$ ) and

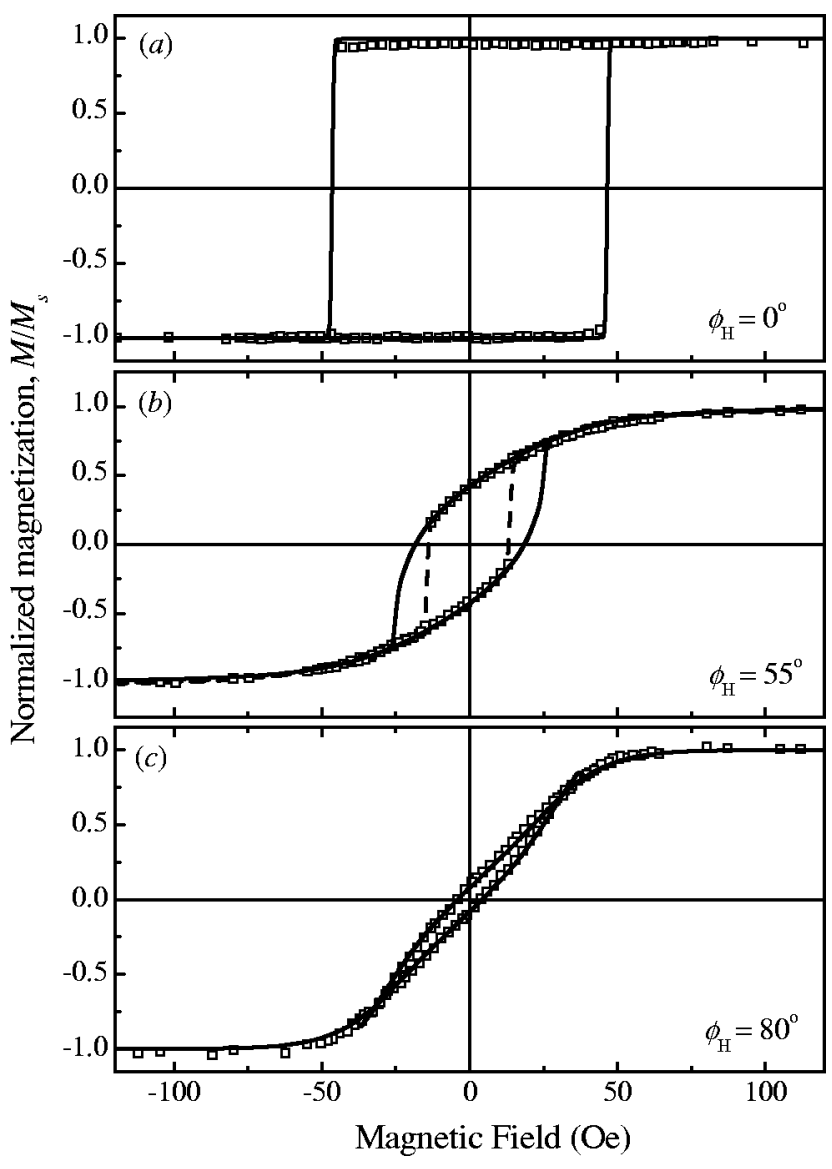

FIG. 3. Experimental in-plane hysteresis loops (symbols) and the corresponding calculated curves for the $\mathrm{Fe}(111)$ film with surface topology shown in Fig. 1(d) with easy axis along the $[1 \overline{1} 0]$ direction, for (a) $\phi_{H}=0^{\circ}$ (i.e., field applied along the [1 $1 \overline{1} 0]$ direction), (b) $\phi_{H}=55^{\circ}$ (the dashed lines represent the irreversible jumps in the measured curve), and (c) $\phi_{H}=80^{\circ}$. The parameters used in the calculations are $K_{2}=2.56 \times 10^{4} \mathrm{erg} / \mathrm{cm}^{3}$ and $K_{u}=4.03 \times 10^{4} \mathrm{erg} / \mathrm{cm}^{3}$.

the Zeeman energy terms, respectively. The unit vectors $\mathbf{u}$ and $\mathbf{n}$ represent the direction of the uniaxial anisotropy and the normal-to-the-film direction.

In zero applied field, if $K_{u}=0$, and for magnetization rotation in the (111) plane, it can be easily shown that the anisotropy is given by $K_{2}$ only, with easy axes along the $\langle 110\rangle$ directions for positive $K_{2}$, and along the three projections of the cube axes in the (111) plane for negative $K_{2}$.

For given $M_{s}, K_{1}, K_{2}, K_{u}, K_{d}, \mathbf{u}, \mathbf{n}$, and $\mathbf{H}$, the static equilibrium direction of $\mathbf{M}_{\mathrm{s}}$ can be calculated from Eq. (1) by finding its polar and azimuthal angles in the spherical coordinates for which $E$ is at minimum; the projection of $\mathbf{M}_{\mathrm{s}}$ along the field direction gives the magnetization of the ferromagnet. The energy minimization procedure used here is described in our previous works. ${ }^{11,12}$

In all calculations, the usual room-temperature $K_{1}$ and $M_{s}$ values for $\mathrm{Fe}$, i.e., $K_{1}=4.8 \times 10^{5} \mathrm{erg} / \mathrm{cm}^{3}$ and $M_{s}$ $=1708 \mathrm{emu} / \mathrm{cm}^{3}$, were used, and $\mathbf{n}$ was assumed to be parallel to the [111] direction.

The experimental hysteresis loop shown in Fig. 2(a) was fitted by a curve calculated assuming that the sample consists of 1800 noninteracting (111)-textured Fe particles with random crystalline orientation in the film plane, each character- 
ized by an energy as that given by Eq. (1), with $K_{u}=0$, and an oblate spheroid shape. This is an ellipsoid for which two of the principal axes are equal, say $a=b$, and $c<a$; if $N_{a}$, $N_{b}$, and $N_{c}$ are the particular values of the demagnetization factor for magnetization along the $a, b$, or $c$ axes, then $N_{a}+$ $N_{b}+N_{c}=4 \pi$. The demagnetization anisotropy constant (the anisotropy is an easy-plane one in the present case) is of the form $K_{d}=\frac{1}{2}\left(N_{a}-N_{c}\right) M_{s}^{2}$, where $N_{c}>N_{a}$.

The best fitting curve, shown in Fig. 2(b), was obtained for $K_{2}=2.56 \times 10^{4} \mathrm{erg} / \mathrm{cm}^{3}$ (i.e., $K_{2} / K_{1}=0.053$ ) and $K_{d}$ $=-2.96 \times 10^{6} \mathrm{erg} / \mathrm{cm}^{3}$, which gives $N_{c}=5.54$. Note that in this case of particulate assembly, the magnetization rotation does not necessarily occur in the (111) plane, and the $K_{1}$ term must be taken into account in the calculations. Using the equations of Osborn, ${ }^{13}$ one can estimate that the former value of $K_{d}$ corresponds to a demagnetizing anisotropy constant of particles with axial ratio $c / a=0.68$, which is in a very good concordance with the mean height-to-width ratio of $0.70 \pm 0.05$ obtained from the STM analysis of the "particles" of our sample; thus, the number of fitting parameters used can actually be reduced to only one, i.e., $K_{2}$. As can be seen, there is rather good agreement between experimental and calculated data for this magnetically isotropic sample. The only noticeable discrepancy is that the squareness of the experimental loop is slightly higher than the model one, which has been assigned to intergranular interactions, ${ }^{14}$ which were not taken into consideration in our calculations.

As mentioned herein, the sequence of hysteresis loops shown in Fig. 3 indicates that the uniaxial in-plane anisotropy of the iron stripes determines the anisotropy of this continuous film. Along with the experimental curves, there are also plotted the corresponding calculated curves for three representative $\phi_{H}$ directions; in this case, the anisotropic part of the free energy is given by Eq. (1) as well. The best results were obtained for the same $K_{2}$ value as the one used to fit the data in Fig. 2(a), and also for $K_{u}=4.03 \times 10^{4} \mathrm{erg} / \mathrm{cm}^{3}$ (i.e., $K_{u} / K_{2}=1.574$, which means that the uniaxial anisotropy is predominant). The unit vector $\mathbf{u}$ is considered to be parallel to the $[1 \overline{1} 0]$ direction, as suggested by the surface analysis results shown in Fig. 1. Due to the high value of the demagnetization (shape) energy constant $\left(K_{d}=-2 \pi M_{s}^{2} \simeq-1.83\right.$ $\times 10^{7} \mathrm{erg} / \mathrm{cm}^{3}$ ), the rotation occurs in the (111) plane; as a consequence, the first term in Eq. (1) is isotropic, so the value of $K_{1}$ does not influence the calculations, as mentioned herein.

It is seen that there is excellent agreement between the experimental and the calculated data. The single apparent difference is the earlier irreversible magnetization jump at the coercive field $(\simeq 14 \mathrm{Oe})$ in the experimental curve as compared to the model one. This is here attributed to formation of a domain wall, with thicknesses $d$ and energy per unit area $\varepsilon$, followed by a rapid domain-wall unwinding. Most probably, this is a Néel wall (with energy $\varepsilon_{N}$ ) which occurs in very thin films due to the very high demagnetization field, and is characterized by the fact that the magnetizations in the wall turn about an axis perpendicular to the plane of the film. ${ }^{15}$

Why is a domain-wall formed and whi is a wall motion observed for $\mathbf{H}$ applied in between the easy and the hard magnetization directions and not for $\mathbf{H} \| \mathbf{u}$ ? In order for a domain wall to be formed, it must be energetically more favorable for the spins to change their directions gradually rather than to have all moments rotating coherently, i.e., to have all spins parallel. It can be demonstrated that for the case considered here, at least up to the coercive field value, $\varepsilon_{N} / d$ is always higher than $E$, the latter being the energy corresponding to the case when all moments rotate coherently [Eq. (1)]. The calculation procedure and the energy expression used are given in the Appendix. For $t=6.0$ $\times 10^{-8} \mathrm{~cm}, \quad \phi_{H}=0^{\circ}$ (field along the easy direction), $M_{s}=1708 \mathrm{emu} / \mathrm{cm}^{3}$, exchange constant $A=1.97$ $\times 10^{-6} \mathrm{erg} / \mathrm{cm}^{3}, \quad K_{2}=2.56 \times 10^{4} \mathrm{erg} / \mathrm{cm}^{3}, \quad$ and $K_{u}=4.03$ $\times 10^{4} \mathrm{erg} / \mathrm{cm}^{3}$, the domain-wall thicknesses is estimated as $d \simeq 2.83 \times 10^{-5} \mathrm{~cm}$ (i.e., $d \gg t$ ), and the corresponding $180^{\circ}$ domain-wall energy per unit area is $\varepsilon_{N}\left(180^{\circ}\right)$ $\simeq 6.65 \mathrm{erg} / \mathrm{cm}^{2}$. In this particular case of $\mathbf{H} \| \mathbf{u}, \varepsilon_{N}$ does not depend on the field, contrary to $E$ which, up to the irreversible magnetization jump in the second and third quadrant, for example, is a linear function of $H$ (negative), $E=-M_{s} H$. Thus, one can estimate the critical field $H_{\text {crit }}$ above which $E$ becomes higher than $\varepsilon_{N}\left(180^{\circ}\right) / d$ from the equality $-M_{s} H=\varepsilon_{N}\left(180^{\circ}\right) / d$, which gives $H_{\text {crit }}$ $\simeq-138$ Oe. Even if the wall is a cross-tie one, ${ }^{16}$ which has an even lower domain-wall energy, i.e., $\varepsilon_{\mathrm{ct}} \simeq 0.6 \varepsilon_{N}\left(180^{\circ}\right)$, the corresponding critical field is $\simeq-83$ Oe. The absolute values obtained for the critical fields are higher than the coercive field of 46 Oe for the case of coherent rotation, so this latter magnetization mode will occur.

The numerical calculations of the fitting curve in Fig. 3 (b) indicate that the measured irreversible magnetization jump takes place between two energy states with an equilibrium angle difference of $\Delta \theta \simeq 150^{\circ}$; therefore, a $150^{\circ}$ Néel domain wall could be formed. In this case, it is much more difficult to estimate $H_{\text {crit }}$ because of the dependence of the Néel domain-wall thickness and energy on $H$; another obstacle for the calculations of $\varepsilon$ is that $\mathbf{H}$ does not lie exactly in the middle between the equilibrium angles. $E$ is not a linear function of $H$ as well. In any case, however, $\varepsilon\left(150^{\circ}\right)<\varepsilon\left(180^{\circ}\right), H_{\text {crit }}$ could be rather lower than that for a $180^{\circ}$ case, and the magnetization transition due to domainwall formation and unwinding could be observed.

The fact that the same values for $M_{s}, K_{1}$, and $K_{2}$ were used to fit both the nanoparticle sample and the continuous film magnetization data indicates that the intrinsic (bulk) properties of the deposited material have not been changed for any of the iron growth modes, as compared to the bulk values for $\mathrm{Fe}$.

In summary, the morphology and the magnetic properties of $\mathrm{Fe}$ thin films grown on deliberately modified $\mathrm{Si}(111)$ substrates were studied. The structural analysis indicated two different iron growth modes; the first one resulted in randomly distributed iron grains, and the second one in wingshaped iron islands. These differently grown patterns are directly related to the preparation of the $\mathrm{Si}(111)$ substrate surface, thus demonstrating that the proper choice of the substrate preparation conditions and the magnetic film thickness can lead to the generation of sophisticated magnetic nanostructures. A phenomenological model was used in order to interpret the experimental magnetization data and to derive 
the anisotropy parameters of both types of Fe samples. It was shown that the magnetic response of the continuous film is determined by the uniaxial anisotropy induced by the substrate topology (a terracelike structure), and that of the nanoparticle sample by both cubic (magnetocrystalline) and shape (easy-plane) anisotropies.

\section{ACKNOWLEDGMENTS}

This work has been supported by the Brazilian agencies CNPq and FAPERGS, as well as by CAPES-COFECUB under Grant No. 375/02.

\section{APPENDIX}

In very thin films (i.e., $d \gg t$, where $d$ and $t$ are the domain-wall and film thicknesses, respectively), due to the very high demagnetization field, the magnetization rotation occurs in the plane of the films. For rotation in the (111) plane, the $K_{1}$ term in Eq. (1) is isotropic, and the anisotropic part of the free energy per unit volume becomes

$$
\begin{aligned}
E_{111}= & \frac{1}{108} K_{2}(1-\cos 6 \theta)+K_{u} \sin \theta \\
& -H M_{s} \cos \left(\phi_{H}-\theta\right),
\end{aligned}
$$

where $\theta$ is the angle between the magnetization and a direction normal to the wall and in the film plane. According to Néel, ${ }^{15}$ by taking $\theta=\Delta \theta(x / d)$ for $-d / 2 \leqslant x \leqslant d / 2$ (where $\Delta \theta$ is the maximum spin direction variation in the wall), the domain-wall thickness may be found by minimizing the total free surface energy $\varepsilon_{N}$, given by

$$
\varepsilon_{N} / d=A\left(\frac{\pi}{d}\right)^{2}+\frac{1}{d} \int_{-d / 2}^{d / 2} E_{111} d x+\frac{\pi t}{d+t} M_{s}^{2},
$$

with respect to $d$ for given $A$ (the exchange constant), $M_{s}$, $K_{2}, K_{u}, t, H$, and $\phi_{H}$. By substituting the value of $d$ thus obtained, the corresponding Néel domain-wall energy can be calculated.

Note that the $E_{111}$ expression contains, besides the usual cubic and/or uniaxial anisotropy terms, the Zeeman term, i.e., the influence of the magnetic field, is taken into account as well.

${ }^{1}$ F. J. Himpsel, J. Phys.: Condens. Matter 11, 9483 (1999).

${ }^{2}$ F. K. Men, F. Liu, P. J. Wang, C. H. Chen, D. L. Cheng, J. L. Lin, and F. J. Himpsel, Phys. Rev. Lett. 88, 096105 (2002).

${ }^{3}$ H. J. Elmers, J. Hauschild, and U. Gradmann, J. Magn. Magn. Mater. 221, 219 (2000).

${ }^{4}$ K. Itaya, R. Sugawara, Y. Morita, and H. Tokumoto, Appl. Phys. Lett. 60, 2534 (1992)

${ }^{5}$ U. Neuwald, H. E. Hessel, A. Feltz, U. Memmert, and R. J. Behm, Appl. Phys. Lett. 60, 1307 (1992).

${ }^{6}$ C. P. Wade and C. E. D. Chidsey, Appl. Phys. Lett. 71, 1679 (1997).

${ }^{7}$ M. H. Wang and L. J. Chen, Appl. Phys. Lett. 62, 1603 (1993).

${ }^{8}$ Y.-T. Cheng, Y.-L. Chen, W.-J. Meng, and Y. Li, Phys. Rev. B 48, 14729 (1993).

${ }^{9}$ Films that show alignment along one substrate crystallographic direction as well as $180^{\circ}$ rotated from this direction are frequently said to be epitaxially grown but twinned.

${ }^{10}$ M. Eddrief, Y. Wang, V. H. Etgens, D. H. Mosca, J.-L. Maurice, J. M. George, A. Fert, and C. Bourgognon, Phys. Rev. B 63, 094428 (2001).

${ }^{11}$ M. Cougo dos Santos, J. Geshev, J. E. Schmidt, S. R. Teixeira, and L. G. Pereira, Phys. Rev. B 61, 1311 (2000).

${ }^{12}$ J. Geshev, A. D. C. Viegas, and J. E. Schmidt, J. Appl. Phys. 84, 1488 (1998).

${ }^{13}$ J. A. Osborn, Phys. Rev. 67, 351 (1945).

${ }^{14}$ J.-G. Zhu and H. N. Bertram, J. Appl. Phys. 63, 3248 (1988); H. N. Bertram and A. K. Bhatia, IEEE Trans. Magn. 9, 127 (1973).

${ }^{15}$ L. Néel, Compt. Rend. 241, 533 (1955).

${ }^{16}$ S. Middelhoek, J. Appl. Phys. 34, 1054 (1963). 